\title{
Results of GPS reprocessing campaign (1996-2011) provided by Geodetic observatory Pecný
}

\author{
Jan Douša, Pavel Václavovic \\ Research Institute of Geodesy, Topography and Cartography, \\ Geodetic Observatory Pecný \\ Ústecká 98, Zdiby 25066 \\ jan.dousa@pecny.cz
}

\begin{abstract}
The paper presents the GOP first reprocessing results, which officially contributed to the EPN-repro1 project. It also describes the 15-year GOP cumulative solution providing station coordinates, velocities and their discontinuities over the period of 1996-2011. Repeatabilities estimated from cleaned long-term coordinate time-series reached 1-2 $\mathrm{mm}$ and 4-6 $\mathrm{mm}$ in horizontal and vertical component, respectively. We then showed the exploitation of GOP reprocessing results in the assessment of the EUREF ITRF2005 densification and the latest ITRS realization, ITRF2008. We identified and confirmed the North-South tilt ( $\approx 2$ mas) in the currently available European reference frame based on the EPN cumulative solution updated in GPS week 1600. The study showed a historical development of the tilt and its close relation to a weak velocity datum definition of this realization, which is very important for a long-term datum prediction. Selected EPN station coordinates, velocities and discontinuities of the latest ITRS realization (ITRF2008) were also assessed. Specific problems for some EPN stations were identified in the global reference frame. This emphasized further necessity to check all the stations before their use for datum definition for regional densifications.
\end{abstract}

Keywords: Global Positioning System, permanent network, reprocessing, terrestrial reference frame, cumulative solution, coordinates, velocities, discontinuities

\section{Introduction}

The EUREF (EUropan REference Frame, http://www.euref-iag.org) is one of the subcommisions for regional reference frames of the International Association of Geodesy (IAG). The EUREF's main goal is to define, to realize and to maintain terrestrial and vertical reference systems in Europe. To serve both scientific and practical applications in positioning and navigation, the European terrestrial reference system (ETRS89) has been realized mainly using GPS observations as they became available at the beging of 90th. The EUREF permanent network (EPN, e.g. [1]) was proposed by W. Gurtner in 1995 [2] in order to provide a continuously operating ground-based infrastructure for such realization of ETRS89. Since that time EPN has developed from 40 stations (1996) to about 300 stations today (September, 2012), almost regularly distributed over Europe and the surrounding areas.

In order to handle a unique and common solution for all EPN stations, a distributed processing scheme of GPS data was organized from the beginning. EUREF subnetworks, routinely processed by 17 local analysis centers (LACs) on a weekly basis, contribute to an EPN 
weekly combined product. European terrestrial reference frame (ETRF), which represents a realization of ETRS89, is based on a long-term combination of EPN weekly solutions. Such combination includes the estimation of station coordinate changes per year (later referred simply as 'velocities') in addition to reference coordinates at a central epoch. Together with a global terrestrial reference frame (labeled as ITRF $<$ year $>$ ), which is maintained by the International Earth Rotation and Reference Systems Service, IERS [3], European reference frame $(\mathrm{ETRF}<$ year $>)$ is established based on densification solutions provided by EPN.

During years, however, the GNSS data analysis of global (and regional) networks were affected by different factors due the updates of reference system realizations, processing models, analyses strategies and software packages. Precise products provided by the International GNSS Services (IGS, [4]) - orbits, Earth Rotation parameters and clock corrections - are dependent on the factors available at the time of generating these products. Thus the global products are not consistent over the past period (1996-2012) and, consequently, also the coordinates estimated by the EPN LACs. Inconsistencies in the time series of the coordinates appear whenever reference frame or commonly adopted models were changed. Global reprocessing activities were started by the Potsdam-Dresden Group [5] and later provided also by other analysis centers in IGS-repro1 project [6] coordinated by IGS. The global reprocessing activities included the generation of improved global products labeled as IGS-repro1 products.

In the meantime some LACs of the EPN also started regional reprocessing activities in order to provide initial solutions homogeneous over a whole period, i.e. without changes in reference frames, global products, processing strategy or applied models. A plan of the coordinated reprocessing activity, spanning over period 1996-2009, was agreed at the EUREF LAC workshop in October 2008. The redistribution of EPN stations was organized (not all LACs were able to contribute), historical archive established, benchmark campaign prepared and evaluated and processing strategies agreed. The solutions of individual subnetworks were then finished in 2011 and combined EPN weekly solutions prepared. The densification of up-to-date global terrestrial reference frame (ITRF2008) could be thus based on a long-term combination of all European stations.

The Geodetic observatory Pecný (GOP) has contributed to EPN as a EUREF local analysis center since January 1997, see [7]. In 2010-2011, GOP LAC processed an extended subnetwork for the EPN reprocessing project. This paper describes daily/weekly GOP solutions and a long-term cumulative (Sec. 2). The GOP-repro1 results were then exploited in the assessment of European ITRF2005 densification based on the EPN cumulative solutions (Sec. 3) and in the assessment of station discontinuities available from the latest terrestrial reference system realization, ITRF2008 (Sec.4). Section 5 sums up with concluding remarks.

\section{GOP-repro1 solution}

This section gives a brief summary of the GOP-repro1 solution which was prepared using the Bernese GNSS software V50 [8] and an in-house developed processing engine. At the first level the processing was carried out by analysing daily data batches and followed with combinations of daily solutions into weekly ones. Figure 1 shows the EPN stations processed by GOP for the EPN-repro1 project. The original subnetwork (stations marked in black) was extended to support the EPN-repro1 requirements that each station is processed by three analysis centres at least. 


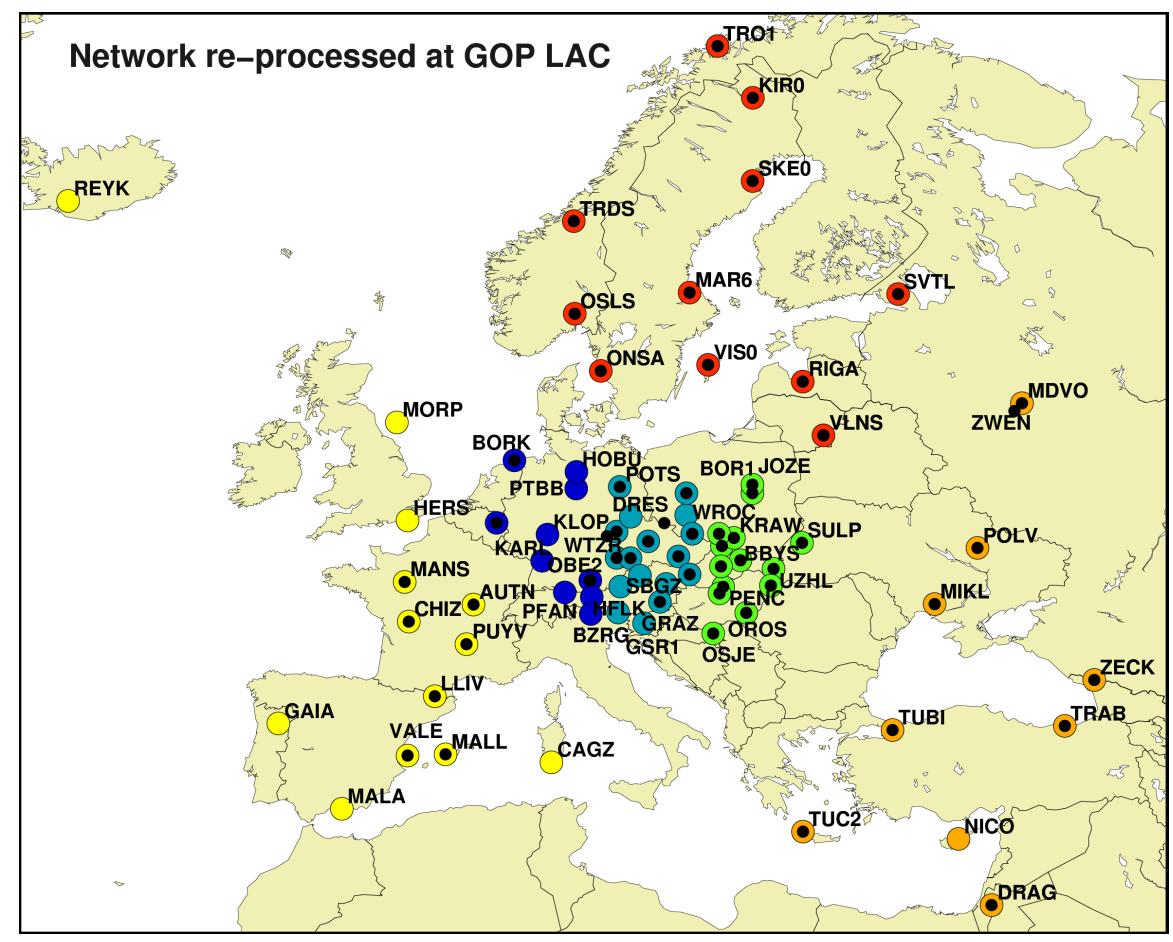

Figure 1: Map of GOP subnetwork extended for the EPN-repro1 project. Different colors shows processing clusters and black color shows the stations from the routine GOP contribution to EPN.

\subsection{Processing strategy}

The global IGS-repro1 products (orbits and Earth rotation parameters) were estimated using the IERS 2003 Conventions [9]. Fixing these global products in regional daily reprocessing, consistent conventions and models needed to be applied, which was followed in most cases, but these two: (1) models were not supported by the latest release of the software package (e.g. the newest tropospheric models in the Bernese GNSS software V5.0), (2) individual antenna calibration models used in EPN, while the IGS follows type-specific models only.

The daily processing consists of the following steps - converting input data and products; preparing a priori coordinates and information; synchronizing receiver clocks; generating single differences over baselines while keeping a maximum number of observations; cleaning data, detecting cycle slips (based on triple-difference approach) and setting ambiguities; screening post-fit residuals and detecting outliers; selecting fiducial stations for datum definition; resolving integer ambiguities; generating ambiguity-fixed daily solutions. The weekly products then include combinations of correspoding daily solutions based on a normal equation stacking procedure. The tropospheric products are finally generated for each day of a week based on a fixing weekly coordinates in new daily adjustments.

The success of resolved integer ambiguities using the QIF strategy [10] is shown in Figure 2. It is strongly seasonally dependent, however, a significant decrease of the percentage of fixed ambiguities is also visible during 1997-2003 which could be partly attributed to the high solar activity (with maximum in 2000) and partly to the lower quality of GPS observations and 


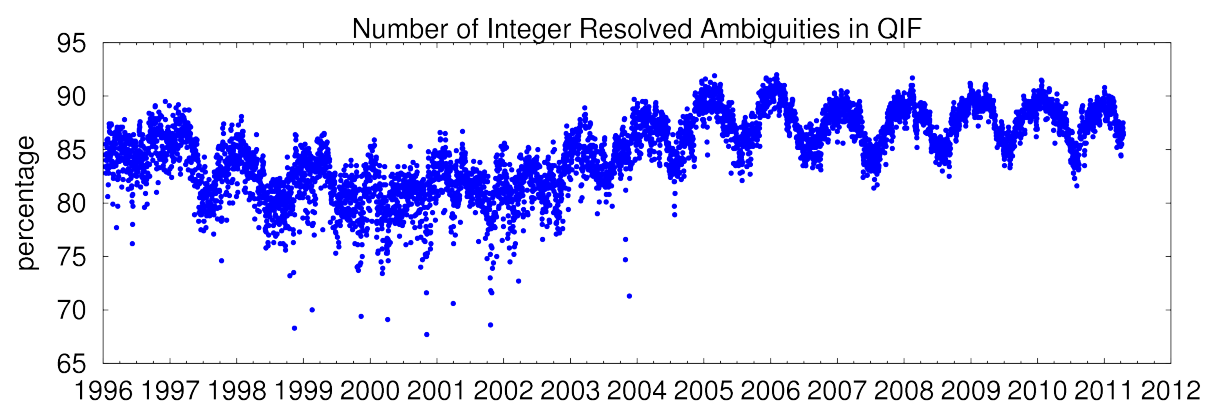

Figure 2: Success of integer ambiguity resolution over processed period

products before 2000. The results of daily and weekly solutions (normal equations) providing station coordinates were archived in the SINEX (Solution INdependent EXchange) format in order to provide official contributions to EPN-repro1 project and in Bernese binary (NQ0) format to support GOP long-term stacking of weekly normal equations.

\subsection{Daily and weekly coordinate time-series}

Daily and weekly solutions provide estimated coordinates of all processed stations in epochs of minimum variance (in ideal case equal to a central epoch of processed data period). Because the subnetwork has a regional extent only, a no-net translation (NNT) condition for a priori coordinates of selected fiducial stations was applied for datum definition. For each daily and weekly solutions, the individual set of consistent fiducial stations was iteratively selected from the a priori list of reference stations. This enabled us to visualize coordinate timeseries in a single geodetic datum and to identify immediately most of the problems related to indidivual station performances. Figure 3 gives only a few examples for six stations: (a) GOPE - instrumentation change, (b) HFLK - seasonal variation probably due to a cumulating snow/ice on top of the antenna radome, (c) OBE2 - a poor data quality, (d) DRAG - station outside the Euroasian tectonic plate, (e) TUBI - non-linear post-seismic station movement and (f) MAR6 - post-glacial uplift in Fennoscandia. These plots were useful for the preparation of GOP long-term cumulative solution and in particular for the definition and revision of station coordinate and velocity discontinuities.

\subsection{GOP long-term solution (1996-2011)}

A 15-year solution was based on a cumulative combination of weekly normal equations, where only coordinates were kept in normal equations. The tropospheric parameters and ambiguities were pre-eliminated during weekly combinations. However, we cannot keep the coordinates constant over a long-term period and we need to apply at least a linear model (i.e. velocity) for each coordinate component. In some cases where non-linear trend in individual coordinate time-series exists (e.g. TUBI in post-seismic period, see Figure 3), a piece-wise linear model need to be applied.

The velocity parameters for all stations are thus introduced into the normal equations just before stacking weekly solutions and generating a unique cumulative one. The velocities can be estimated thanks to the applying long period during which the coordinates significantly 

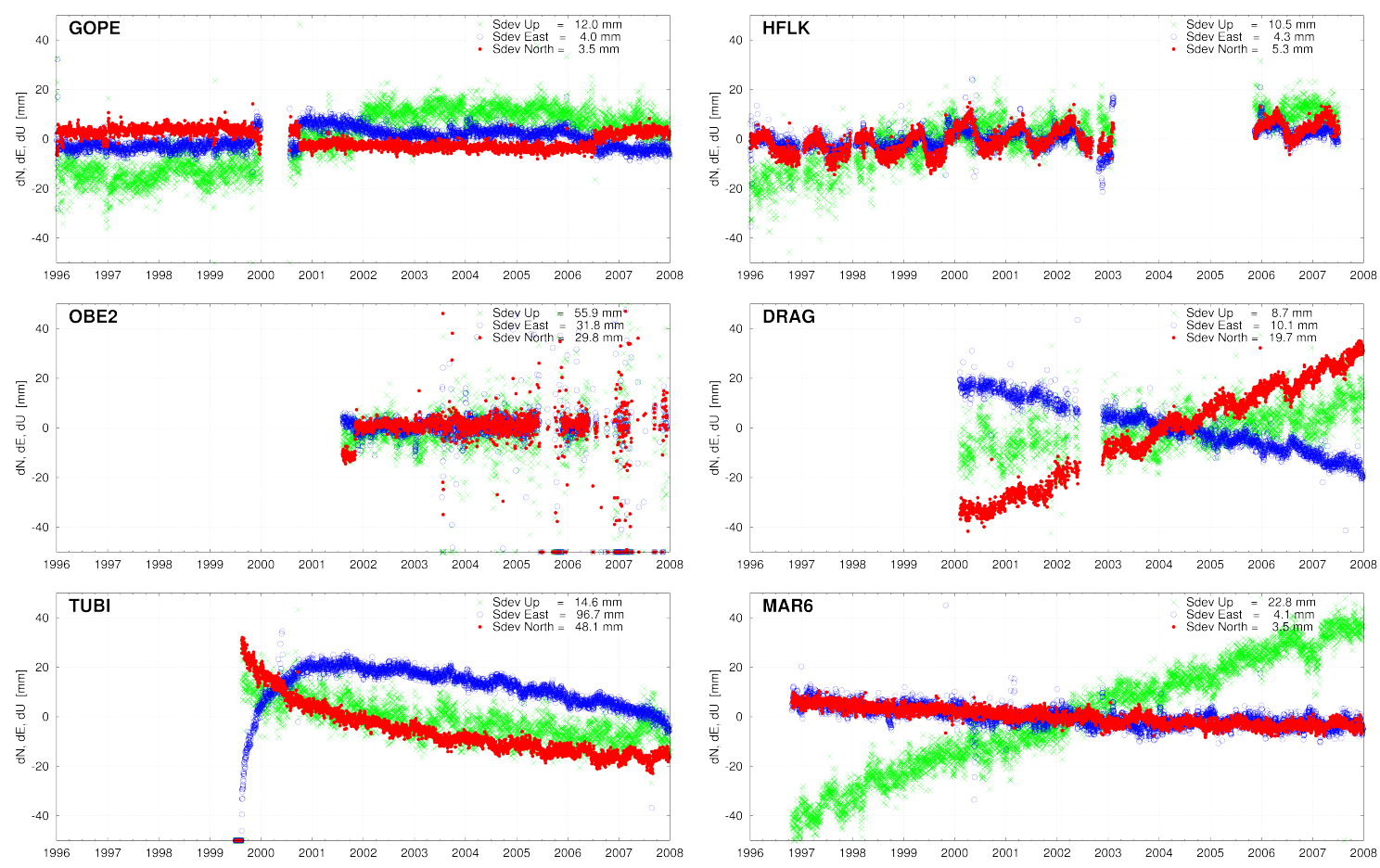

Figure 3: Example of coordinate time-series for six EPN stations from GOP daily solutions
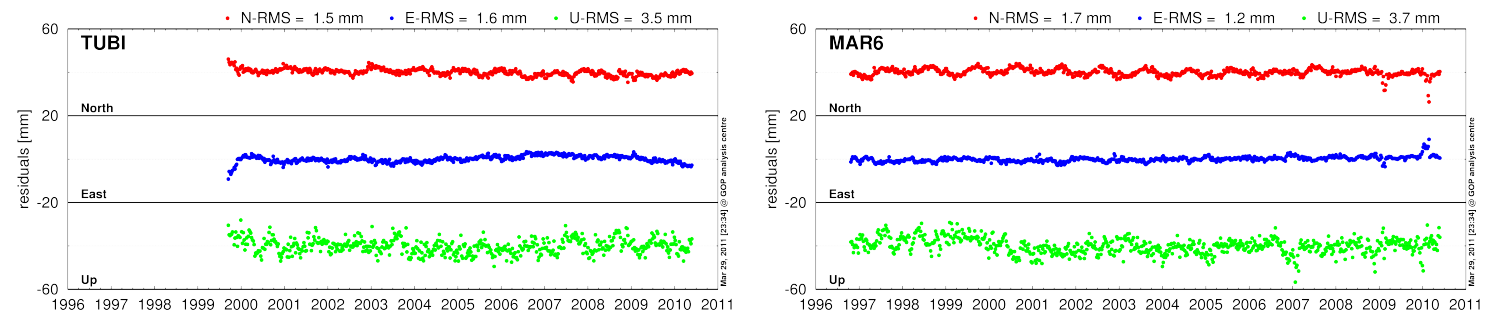

Figure 4: Cleaned coordinate time-series from the final cumulative solution

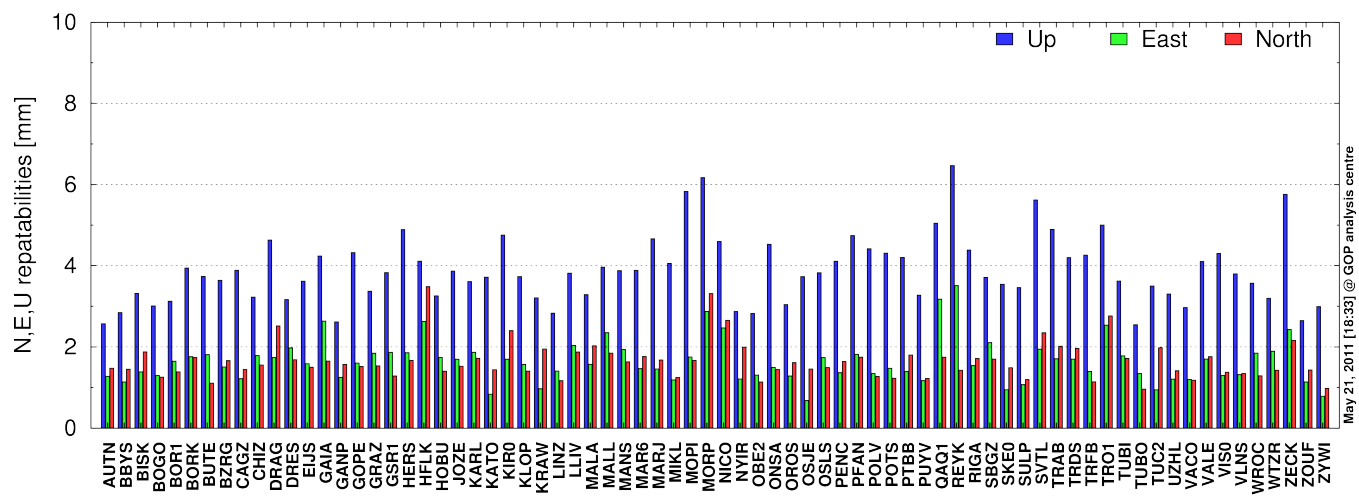

Figure 5: Coordinate repeatabilities of the GOP final cumulative solutions 


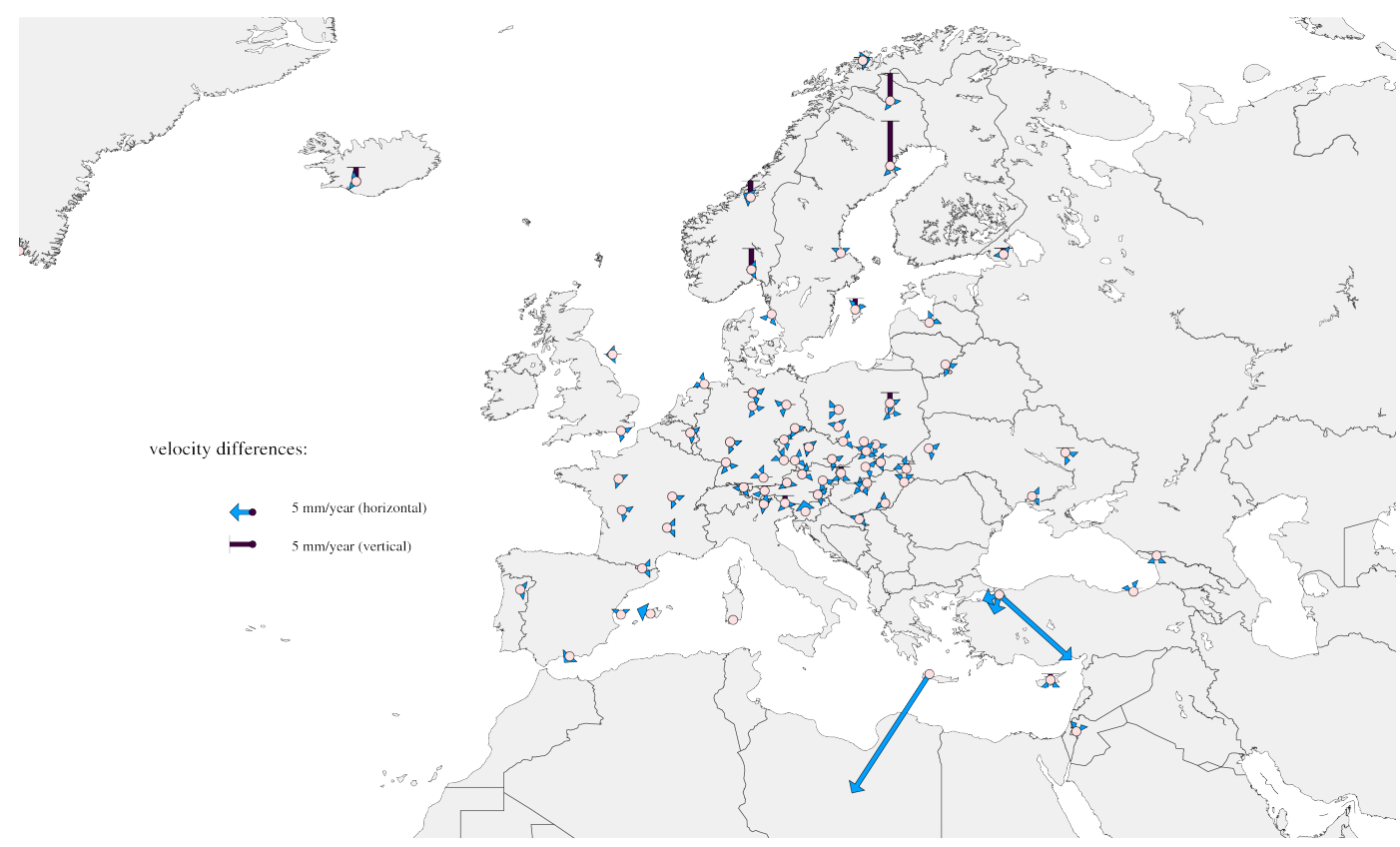

Figure 6: Residuals velocities with respect to ITRF2005

change (decorrelating the velocity estimation from the coordinates). For the stations with too short periods (usually less than a year), the velocities could be either constrained to some well-known a priori values or they are estimated with large formal errors showing correctly large uncertainties. A long-term solution was prepared based on the folowing steps, which might be individually re-iterated if necessary:

- $1^{\text {st }}$ step : check residuals and indentify bad data periods

- $2^{\text {nd }}$ step : setup discontinuities for coordinates and velocities

- $3^{\text {rd }}$ step : select the best set of fiducial stations and provide final solution

A final solution is achieved after a careful cleaning of all poor data (usually identified as outliers or based on other external knowledges) and after setting discontinuities for all cases when station coordinates or velocities show significant jumps. Cleaned coordinate time-series (Figure 4) are results of such final solution, which already provides homogeneous repeatabilities for all station coordinates (Figure 5). In our solution, the repeatability was 1-3 mm and 4-6 $\mathrm{mm}$ for the horizontal and vertical component, respectively. The highest repeatabilities are clearly found at the station HFLK (on top of the Alp mountain) and those stations located at the margins of the network (stations QAQ1, REYK, MORP, TRO1, KIR0, SVTL, DRAG, NICO, TRAB, ZECK). This is due to their isolations (long-baselines) as well as less contributing observations. The datum of the GOP final solution was defined using a set of 18 stations. The maximum residuals after applying Helmert transformation (using translations only), between a priori and estimated coordinates, were below $5 \mathrm{~mm}$ in horizontal and $15 \mathrm{~mm}$ in a vertical components.

The estimated velocity differences with respect to the motion of the Eurasian tectonic plate (Figure 6) demonstrated stable changes of coordinates for most of the stations (usually below $1 \mathrm{~mm} /$ year). This indicates a stability of the tectonic plate with respect to some others in 

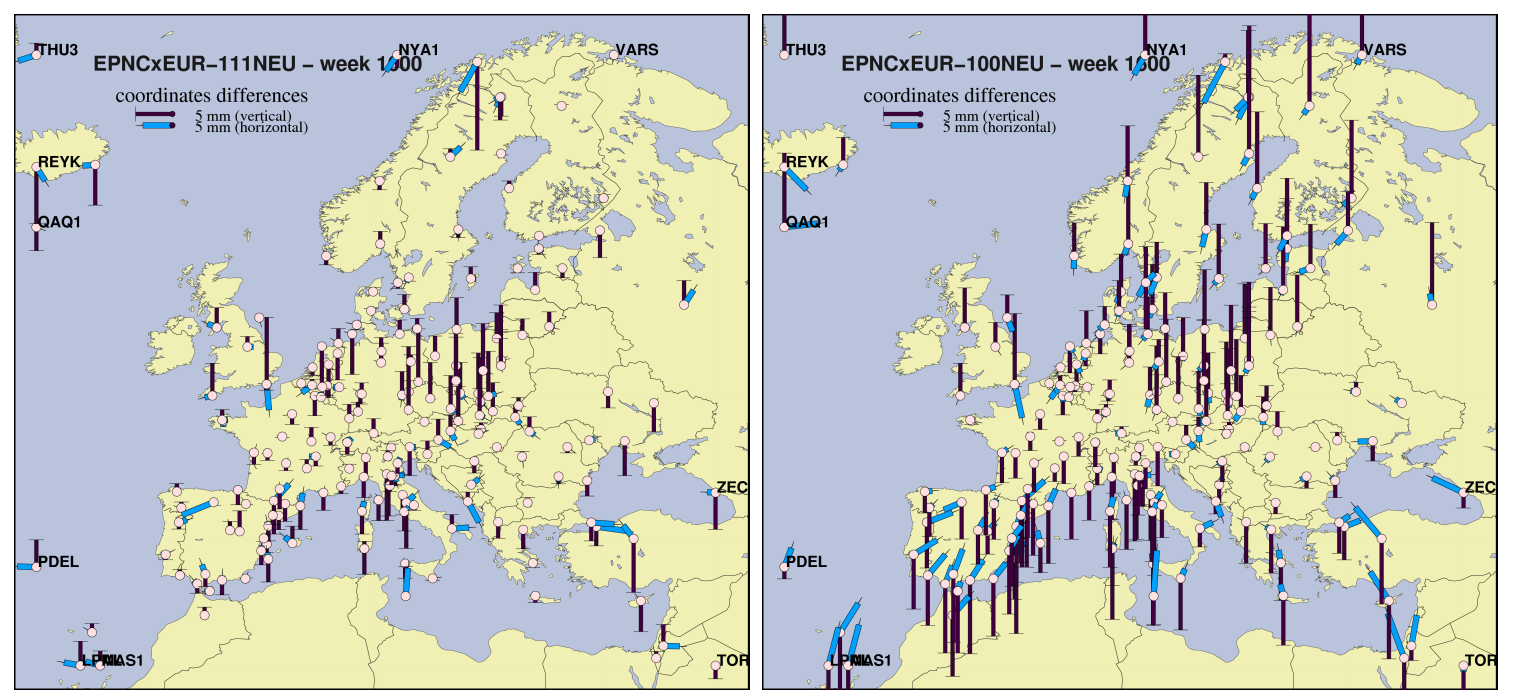

Figure 7: Residuals from the Helmert transformation at epoch of September, 7, 2010 (GPS week 1600) between the EPN cumulative solution (EPNC, updated at GPS week 1600) and EPN weekly solution (1600). Left: 7 parameters; Right: 3 translations (translation).

the world. However, we could clearly observe such geophysical phenomenas like: (a) postglacial uplift in Fennoscandia, which is of different magnitude for various stations, (b) a single station in Greece representing a drift in the South-West direction common to most of the Greek islands, (c) non-linear post-seismic relaxation (modelled by piece-wise constant velocities) for TUBI station after the Izmit earthquake in 1999 (Mw 7.4).

\section{Evaluation of European ITRF2005 densification}

On November 5 2006, the IGS adopted new reference frame (ITRF2005) and new model for antenna phase center offsets and variations (PCV, IGS08) for its global orbit and clock products. This change caused jump at that epoch for almost all EPN station coordinates. The velocity estimation should not be teoretically affected if discontinuities are correctly setup and thus only velocities could provide a datum for the prediction of coordinates over a period of 3-6 years later (i.e. before a new ITRS realization was available).

The preparation and maintenance of the ETRS89 realization is one of the tasks of the EUREF Technical Working Group (TWG). Any further densification of ETRS89 on national level is also usually validated by the EUREF TWG. During 2010, several national densifications campaigns were validated: EUREF-2009-IR/UK, EUREF-2010-Czech and EUREF-2010-Serbia. Except EUREF-2010-Czech, which concerned a solution over three years [11], the others showed a North-South tilt from the coordinate residuals of Helmert transformation when applying (a) 3 translations +3 rotations + scale or (b) 3 translations only. The reason was not known at that time, but the first impression was that the problem was not related to the campaigns themselves, but to the cumulative EPN solution providing a last realizion of the ETRS89. Consequently, an action item was raised to identify this problem.

We could use the GOP-repro1 as an existing historical homogeneous solution for more deep study, however, at the first stage we tested for which EPN stations the North-South tilt can be 


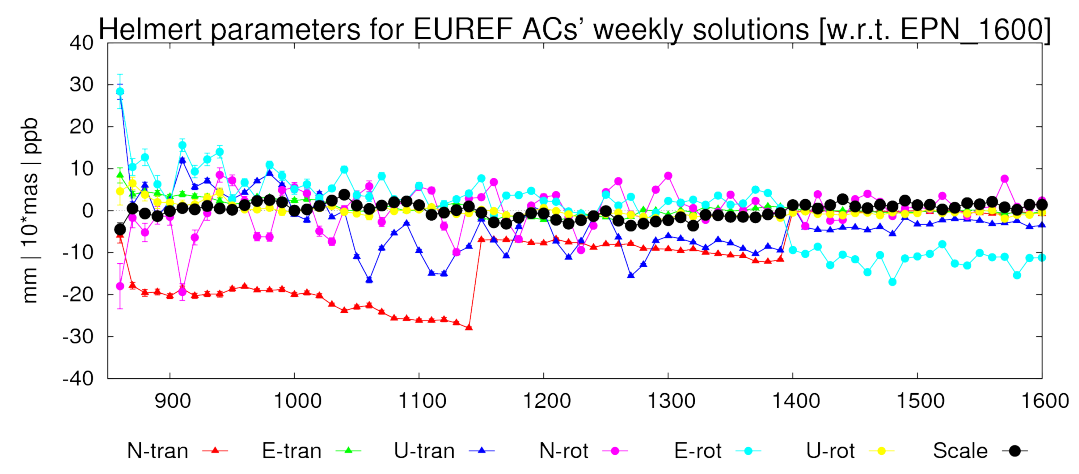

Figure 8: time-series of estimated Helmert parameters between EPN weekly and EPN cumulative solutions. All stations used; x-axis represents time in GPS week.

observed. We compared one of the latest combined weekly EPN solutions with respect to the latest update of the EPN cumulative solution. We used GPS week 1600 and all EPN active stations from which we iteratively selected a set of 'consistent' stations to be used as fiducials for the Helmert transformation. We applied 3 (translations) and 7 (translations, rotations and scale) parameters in the transformation between coordinates at a reference epoch in the middle of GPS week 1600 (September 8, 2010). Figure 7 shows the results, which clearly confirmed a common North-South tilt over all stations between the two solutions of about 2 mas (and additionally a small scale difference). This corresponds to about $\pm 1 \mathrm{~cm}$ for heigh component for stations in North and South Europe for given epoch. Our expectation that the problem exists in the cumulutive solution was confirmed.

We have then run a similar test for every solution over a whole EPN period of GNSS observations (1996-2010) with a 10-week step. We should emphasize that the EPN cumulative solution provided coordinates, velocities and discontinuities at the common central epoch 2000.0, while the EPN weekly solutions provided coordinates at a central epoch of each week. Before applying Helmert transformations the coordinates of the EPN cumulative solution were firstly converted (by applying velocities) to the central epoch of EPN weekly solution. In order to provide a robust estimation of Helmert parameters, all available EPN stations were set a priori as fiducials while during an iterative procedure all those not consistent were temporarily excluded. Discontinuities defined in the EPN cumulative solution was necessary to take into account as well as to apply renaming scheme to compare theoretically identical stations only.

Time-series of 7 Helmert parameters between the two coordinate sets are depicted in Figure 8. The figure clearly shows jumps in the time-series due to existing inconsistencies in EUREF weekly solutions over the entire period. The inconsistencies are related to reference frame changes and different models applied (e.g. at GPS week 1400 antenna PCV model was switched from relative to absolute calibrations). The figure also shows time-series of Helmert parameters estimated in a local reference system centered at the geometrical centre of the EPN network. The North-South tilt, which is represented by the rotation around the East axis in the local system, has been clearly observed since GPS week 1400. In the global system the tilt is represented by X- and Z-translations and Y-rotation (not shown here). The figure was important to prove that we are able to reconstruct Helmert parameter time-series estimated 


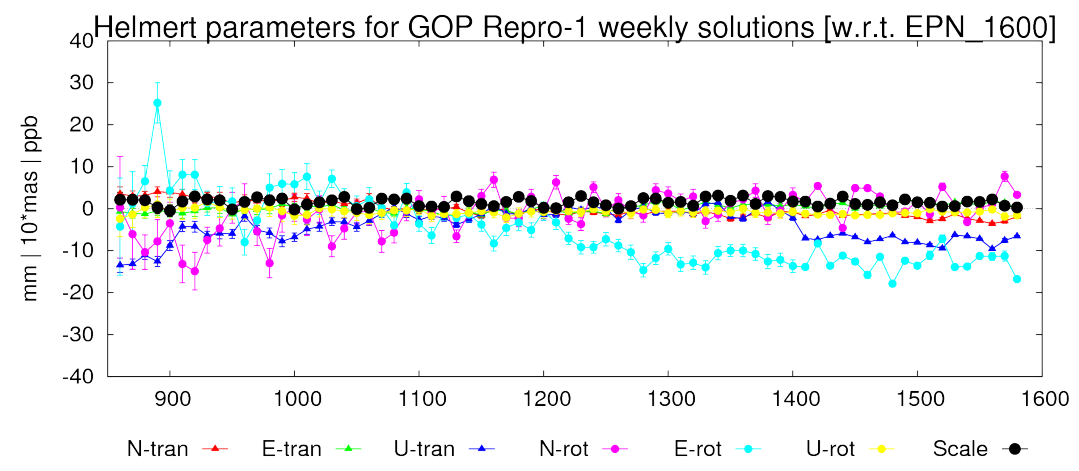

Figure 9: time-series of estimated Helmert parameters between GOP-repro1 and EPN cumulative solutions. GOP-repro1 stations used only; x-axis represents time in GPS week.

by the CATREF software [12] used during the combination of EPN weekly solutions into a cumulative one.

Additionally the figure demonstrated that our strategy developed for automated selection of fiducials was enough robust to achieve the same results as with datum uniquely defined during the EPN cumulative solution. The same strategy could be then used for the estimating tranformation parameters for GOP-repro1 weekly solutions with respect to the EPN cumulative solution and plotting corresponding time-series. Being a homogeneously reproccessing the GOP-repro1 solution enabled to reconstruct a full history of estimated Helmert parameters in a consistent way. Figure 9 shows such smooth time-series not affected by regular updates of reference frame or antenna calibration model.

The GOP-repro1 weekly products didn't include all EPN stations, but well covered the territory of Europe. We could guarantee the possible replacement of a complete EPN-repro1 solution, which was not available at that time, with the GOP-repro1 since reaching a good agreement of Helmert parameters estimated for both solutions after GPS week 1400. All comparisons before GPS weeks 1400 for GOP-repro1 (Figure 9) thus represents a successful reconstruction of the full historical development of Helmert parameters and also the development in the trend of the North-South tilt. From the clear linear trend, the tilt can be understood as a product of the weak velocity datum definition in original and European ITRF2005 densifications. And this further resulted in the North-South tilt increasing with the lenght of the coordinate prediction.

\section{ITRF2008 discontinuities assessed using GOP cumulative solutions}

The GOP-repro1 cumulative solution gave us an opportunity to assess the latest ITRS realization, ITRF2008. The motivation was raised again during the validation of national ETRS89 densification campaigns. The question was wheather the station REYK can be used as fiducial due to a significant coordinate jump in the ITRF2008. Such jump was, however, not visible in the EPN cumulative solution although an earthquake occured on September 11, 2008 close to the station.

The GOP-repro1 proved that the REYK position was not disrupted by the earthquake and relevant discontinuity in the coordinate time-series is not necessary. For visualization, we have 
developed a plot schemes showing clearly all instrumentation and reference frame changes at each station. Additionally, these plots include all setup discontinuities (coordinate offsets) as they were estimated in various reference frame realizations. Finally, the GOP-repro1 cumulative solution residuals are plotted to show all remaining (unmodeled) effects in the coordinate time-series. Figure 10 shows an example for station REYK with the specific jump (September 11, 2008) in coordinates from ITRF2008. The GOP-repro1 residuals before and after this epoch are smooth although a discontinuity was not setup for this epoch. Another station showing unrealistic discontinuities of several centimeters was TRDS (Figure 11) from those included in GOP-repro1 solution.

Besides discontinuities and their comparisons the plots clearly display the inconsistencies in station availability in various solutions. A limited validity of a coordinate prediction exists whenever the discontinuities were recently defined and observations from later period were not available. Figure 10 (REYK) and Figure 11 (TRDS) can represent typical, but not worst examples. Other examples are MAR6, RIGA and JOEN (not shown) providing an uninterrupted coordinate time-series over a whole period, but with missing first three years in ITRF2008.

The above assessments were important to demonstrate the necessity of the careful ITRF2008 station selection for their use as fiducials for regional or national densifications. It also shows some weak points of a global GNSS solution when considering aspect of regional permanent stations. The IGS-repro1, which was a basis for a global GNSS contribution to the ITRF2008, didn't provide the best solutions for at least the following cases: (1) for stations with missing data when compared to their official validity (and availability) within the EPN or, opposite, stations with data excluded from this period (both affecting the quality of estimated velocities) (2) for stations which discontinuties were not properly handled and, consequently, the station can not be considered as fiducial for this period or later (meaning that coordinates for specific periods were not correctly estimated).

\section{Conclusion}

The paper presented the GOP first reprocessing results, which officially contributed to the EPN-repro1 project. Additionally, the 15-year GOP cumulative solution was described providing long-term station coordinates, velocities and their discontinuity estimation. Repeatabilities estimated from cleaned long-term coordinate time-series reached 1-2 $\mathrm{mm}$ and $4-6 \mathrm{~mm}$ in horizontal and vertical component, respectively. We then showed the exploitation of GOP reprocessing results in the assessment of the EUREF ITRF2005 densification and the latest ITRS realization, ITRF2008.

We identified and confirmed the North-South tilt $(\approx 2 m a s)$ in the currently available European reference frame based on the EPN ITRF2005 densification solution (e.g. last EPN cumulative solution updated in GPS week 1600. The study showed a historical development of the tilt and its close relation to a weak velocity datum definition in this frame. The velocity datum is particularly important for a long-term datum prediction when for most stations discontinuities were setup due to changes in processing strategy. The results of the tilt study were presented at the EUREF Technical Working Group meeting (Padua, March 2011) and confirmed by new European ITRF2008 densification already based on solutions from the EPN-repro1 project. 
REYK discontinuities/residuals - ITRF2005, ITRF2008, EPN_Cummul, GOP_Repro1

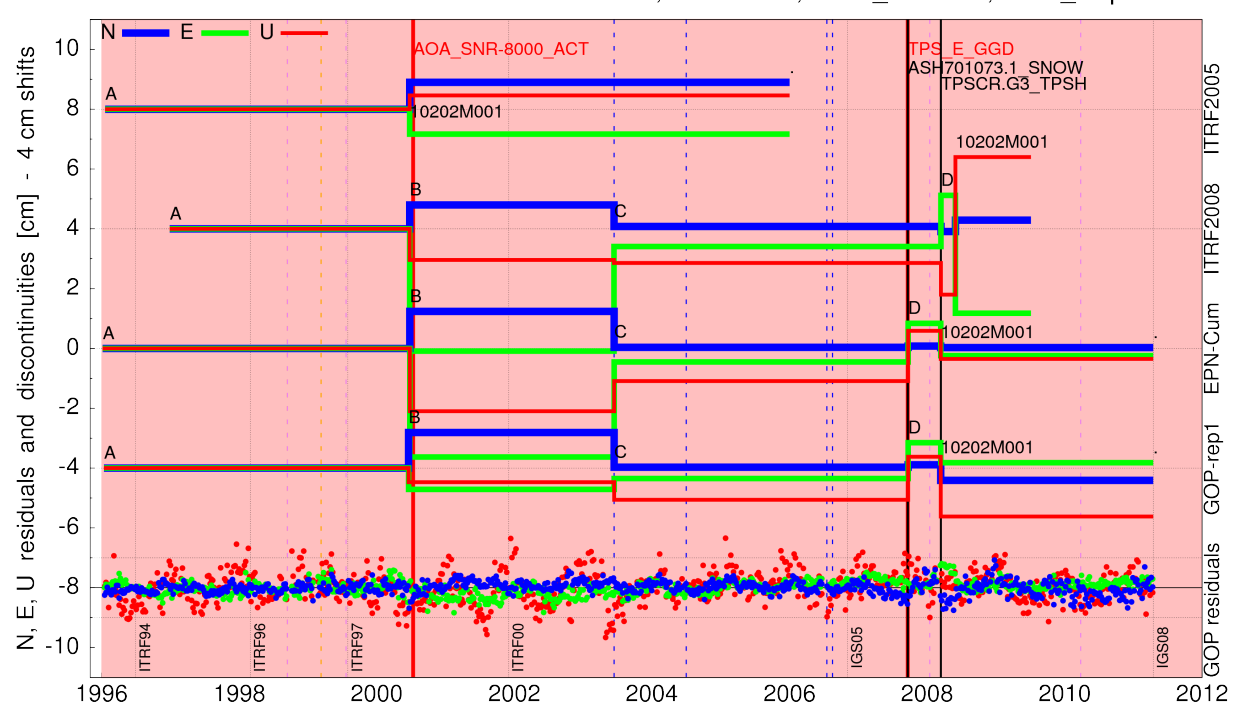

Figure 10: Coordinates discontinuities for station REYK for various solutions (ITRF2005, ITRF2008, EPN cumulative solution updated in 1600 and GOP-repro1 for which also residuals are shown). The changes in reference frames are marked in magenta besides the station specific instrumentation updates.

TRDS discontinuities/residuals - ITRF2005, ITRF2008, EPN_Cummul, GO0_105-C

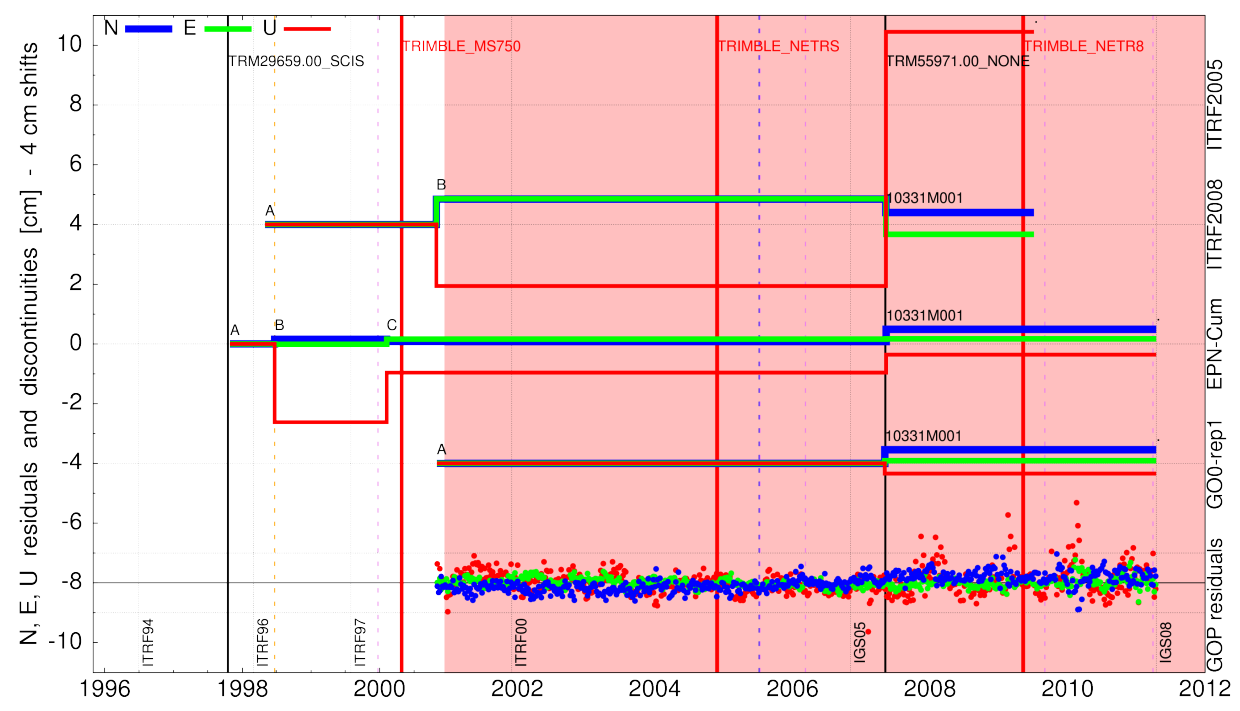

Figure 11: Coordinates discontinuities for station TRDS for various solutions (ITRF2005, ITRF2008, EPN cumulative solution updated in 1600 and GOP-repro1 for which also residuals are shown). The changes in reference frames are marked in magenta besides the station specific instrumentation updates. 
Finally, selected EPN station coordinates, velocities and discontinuities of the latest ITRS realization (ITRF2008) were assessed using GOP-repro1 results. Specific problems for some EPN stations were identified in the global reference frame. This emphasized a further necessity of careful revision of all ITRF2008/IGS08 stations before their use for datum definition in regional densifications.

\section{Acknowledgements}

This work was partly supported by the Technology Agency of the Czech Republic (TB01CUZK006) and by the European Regional Development Fund (ERDF), project 'NTIS - New Technologies for Information Society', European Centre of Excellence, CZ.1.05/1.1.00/02.0090.

\section{References}

[1] Bruyninx C.: The EUREF Permanent Network: a multi-disciplinary network serving surveyors as well as scientists", GeoInformatics, Vol 7, pp. 32-35, 2004

[2] Gurtner W.: Guidelines for a Permanent EUREF GPS Network, In Report on the Symposium of the IAG Subcommission for the European Reference Frame (EUREF) held in Helsinki, 3-5 May 1995, Gubler, E. and H. Hornik (eds), Bayerische Akademie der Wissenschaften, Muenchen, EUREF publ. No 4, pp. 68-72, 1995

[3] IERS - International Earth Rotation and Reference Systems Service, http://www.iers.org

[4] Dow J., Neilan R.E., Rizos C.: The International GNSS Service in a changing landscape of Global Navigation Satellite Systems, J. Geod., Vol. 83, pp: 191-198, (2009), doi:10.1007/s00190-008-0300-3

[5] Steigenberger P., M. Rothacher, R. Dietrich, M. Fritsche, A. Ruelke, S. Vey (2006), Reprocessing of a global GPS network, J. Geophys. Res., Vol. 111, B05402 (2006), doi:10.1029/2005JB003747

[6] IGS-repro1 project: http://acc.igs.org/reprocess.html

[7] Douša, J., Ehrnsperger W.: Permanent GPS Data Processing at the BEK and the GOP EUREF Local Analysis Center, In Report on the Symposium of the IAG Subcommission for the European Reference Frame (EUREF) held in Sofia, 4-7 June 1997, Gubler, E. and H. Hornik (eds), Bayerische Akademie der Wissenschaften, Muenchen, EUREF publ. No 6, pp. 59-63, 1997

[8] Dach R., Beutler G, Bock H., Fridez P., Gade A., Hugentobler U., Jaggi A., Meindl M., Mervart L., Prange L., Schaer S., Springer T., Urschl C., Walser P.: Bernese GPS Software Version 5.0, Astronomical Institute, University of Bern, Bern, Switzerland, 2007, User manual.

[9] IERS Conventions (2003): Dennis D. McCarthy and Gerard Petit (eds.), (IERS Technical Note 32) Frankfurt am Main: Verlag des Bundesamts fuer Kartographie und Geodaesie, 2004, 127 pp., ISBN 3-89888-884-3 (print version)

[10] Mervart L.:, Ambiguity resolution techniques in geodetic and geodynamic applications of the Global Positioning System. Geod. Geophys. Arb. Schweiz, No. 53, p. 155, 1995 
[11] Douša J., Filler V., Kostelecký J.(jr), Kostelecký J., Šimek J: EUREF-Czech-2009 campaign, presented on the Symposium of the IAG Subcommission for Europe (EUREF) held in Gaevle, Sweden, 2-5 June, 2010

[12] Altamimi Z., Sillard P., Boucher C.: CATREF software: Combination and analysis of terrestrial reference frames. LAREG Technical Note SP08, Institut Geographique National, France, 2004. 
